\title{
Vehicle Relocation Triggering Thresholds Determination in Electric Carsharing System under Stochastic Demand
}

\author{
Wanjing Ma $\mathbb{D}^{1},{ }^{1}$ Lei Wang $\mathbb{D}^{1},{ }^{1}$ and Lin Li $\mathbb{D}^{2}$ \\ ${ }^{1}$ The Key Laboratory of Road and Traffic Engineering of the Ministry of Education, Tongji University, Shanghai 201804, China \\ ${ }^{2}$ Shanghai SH Intelligent Automotive Technology Co., Ltd., Shanghai 201805, China \\ Correspondence should be addressed to Lin Li; lilin@siac-sh.com
}

Received 16 March 2018; Revised 31 May 2018; Accepted 13 June 2018; Published 29 July 2018

Academic Editor: Jose E. Naranjo

Copyright (c) 2018 Wanjing Ma et al. This is an open access article distributed under the Creative Commons Attribution License, which permits unrestricted use, distribution, and reproduction in any medium, provided the original work is properly cited.

\begin{abstract}
Vehicle unbalance usually occurs in multistation electric carsharing systems. Threshold triggering method is one of the most practicable approaches for vehicle relocation, while determination of thresholds has not been sufficiently studied particularly for electric carsharing system. This paper presents an approach on determining the thresholds considering the stochastic demands and system states. Firstly, we establish a state transition model involving the stochastic variables to capture the dynamics of the number and battery status of vehicles as well as the traffic demands. Consequently, a dual-objective optimization model was developed to determine the proper values of thresholds. The solution algorithm employed the min-max robust optimization to tackle the uncertainty and the Pareto optimum to decide the solution under dual objectives. To test the distribution stochastic variables, we involve the orders data and the supplementary user survey. Comparison is conducted among three methods: the empirical rules, the deterministic method, and the stochastic method, where the results suggest that the stochastic method achieves better solutions on the dual objectives under stochastic demands.
\end{abstract}

\section{Introduction}

Carsharing mode has obtained extensive attention in either personal-vehicle-oriented or public-transit-oriented cities, including in Europe, North America [1], and some parts of Asia [2]. By 2015, there had been over 1.1 million members and 16,754 vehicles in the US since the first carsharing program launched in 1994 [3]. In China, from the emergence of carsharing services in 2009 to 2015, more than 330,000 people signed up for a carsharing membership, and about 5000 vehicles had been in operation, where half of them were electric vehicles $(\mathrm{EV})$ and five of the eight organizations ran EVs and three of them were EV-only [4].

Traditional carsharing models require users to pick up and return vehicles at the same station and with strictly appointed time (as roundtrip carsharing). In order to increase the accessibility, provide better mobility, attract more users, and strengthen competitiveness, carsharing organizations (CSO) introduced flexible pick-up and drop-off location and time (namely, one-way carsharing) that allows users to pick up a vehicle on demand and return it to other stations without a preconcerted arrangement. It is suggested that oneway carsharing is experiencing a rapid worldwide expansion contemporarily [5], new one-way CSOs are emerging (e.g., Car2Go and DriveNow), and existing CSOs are gradually offering one-way capability in major cities (e.g., Zipcar).

However, accompanying the improvement of the flexibility, the distribution of fleets in one-way models potentially turns to be imbalanced with demands variation. The unbalance of demands and fleets makes it necessary to relocate vehicles for sustainable operation. On the other hand, the on-demand pick-up and drop-off rule also causes the preplanned relocation to be unfeasible, especially under stochastic demands, which requires the relocation mechanisms to take the randomness into account.

The threshold based relocation strategy has been developed to respond to the dynamic system changes $[6,7]$. The thresholds are applied to keep the number of vehicles within a proper range in each station (as shown in Figure 1(a)). This mechanism was also concluded into two major steps (as 

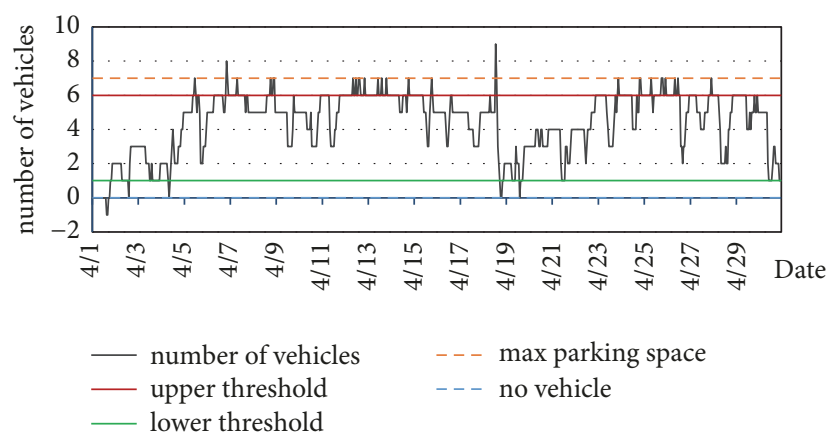

(a) Thresholds to control the number of vehicles changing within a proper range

\begin{tabular}{|l|l|}
\hline \multicolumn{2}{|c|}{ State listening } \\
\hline The number of vehicles: \\
$\begin{array}{l}\text { TIf } \geqslant \text { upper threshold, then tag: move out } \\
\text { - If } \leqslant \text { lower threshold, then tag: move in }\end{array}$
\end{tabular}

(b) Two-step threshold based relocation method

FIGURE 1: The threshold based relocation approach.

shown in Figure 1(b)): (1) The system monitors the change of the number of vehicles (the state variable) in each station. If the number of vehicles of a station accumulated above an upper threshold, tag the station as which needs moving out cars (overflowing); if the number of vehicles of a station decreased below a lower threshold, tag the station as which needs moving in cars (lacking). (2) Relocation listening: The system monitors the relocation tag of each station and decides the optimum solutions of path to move vehicles from overflowing stations to lacking stations, with the constraints of relocation staff resources.

It should be noted that the thresholds setting is a fundamental work of the threshold based relocation mechanism. To the best of our knowledge, there had been some studies based on designated thresholds, but detailed study towards thresholds determination is still needed to be extended.

The main targets of this paper are as follows:

(i) To propose a quantitative method for the triggering thresholds determination

(ii) To capture the uncertainty of demands (the solutions of thresholds are decided through a "min-max" robust optimization to find the best solution in the worst condition)

(iii) To weigh both service quality and the relocation times, applying the Pareto front to find the nondominant sets for both the worst condition stochastic demand sets and the best objectives solution sets

(iv) To involve the battery and charging properties of EV as a major difference with conventional carsharing systems.
The rest of the paper is structured as follows: Section 2 reviews the literature concerning the carsharing vehicle relocation problem; Section 3 is the modeling of station vehicle changing and the robust optimization of thresholds under stochastic demand; Section 4 presents a solution algorithm; Section 5 presents the analysis and discussion; and the last section concludes the study.

\section{Literature Review}

2.1. Study on Vehicle Relocation Problems. Studies on carsharing mode generally fall into two categories: strategy planning level and operating level. Strategy level covers issues of environment and resources benefits $[8,9]$, market investigation $[10,11]$, users' behavior $[12,13]$, and network design $[14,15]$. Operating level problems also received attention, but studies are still not sufficient to support the industrial development, especially for one-way model, which allows users to pick up and drop off vehicles at different stations. As one major difficulty in the system operation, the fleet imbalance problem makes the study on vehicle relocation even more urgent.

Vehicle relocation problem had been studied through many approaches, as what we have summarized in Table 1. For some preliminary one-way carsharing systems which require users to provide reservation information, mathematical programming approaches can be adopted without many obstacles. With demands known, the problem can be formulated as mixed integer programming and efficiently solved by feasible solvers [14, 16-18].

With the deeper penetration of on-demand/instant access one-way demand systems, which allow users to get access to 
TABLE 1: Summary of studies on vehicle relocation of carsharing systems.

\begin{tabular}{lcc}
\hline Types & Approaches & Representative studies \\
\hline $\begin{array}{l}\text { Reservation-based (with demands } \\
\text { known) }\end{array}$ & Mathematical planning & $\begin{array}{c}\text { Nourinejad (2014), Carlier (2015), } \\
\text { Zakaria (2014), Boyac1 (2015) }\end{array}$ \\
\hline & Threshold based & Barth (1999), Kek (2009), Cepolina (2012, \\
2014) \\
$\begin{array}{l}\text { On-demand / instant access (with } \\
\text { demands unknown) }\end{array}$ & $\begin{array}{c}\text { Demand prediction \& mathematical } \\
\text { programming } \\
\text { Rolling horizon \& mathematical } \\
\text { programming } \\
\text { Stochastic programming }\end{array}$ & Bruglieri (2014), Repoux (2015) \\
& Febbraro (2012), Santos (2015) \\
\hline
\end{tabular}

the system without previous reservation, this policy makes the demands unknown. One approach is to predict demands then apply deterministic mathematical programming model $[19,20]$ or to collect short-term demands and then model with rolling horizon scheme [21, 22]. Besides optimization, a practical approach is to dynamically detect the number of available vehicles in stations and trigger relocation tactic by judgment of thresholds. This method has been regarded as an easy way to practice and enables fast implementation for carsharing operators [23].

2.2. Threshold Based Vehicle Relocation Problem. The concept of thresholds was firstly introduced by Barth (1999) [23], which involved a minimum threshold to judge a vehicle insufficiency condition and generate a request to relocate vehicle in. This threshold enables an intuitive vehicle relocation approach which was named as static relocation by that time. There had not been more detailed explanations on mechanism and setting of thresholds. Based on Barth's concept, Kek et al. (2006) [6] further discussed the role of thresholds, which are defined as the upper and lower limits on the number of vehicles in a station. When thresholds are reached, there should be an operation to remove or add vehicles at the station. Kek analyzed the effects of different range of thresholds from a logical perspective but did not quantitatively model the dynamics behind the system. Kek et al. (2009) provided more thinking about thresholds and explained more details of the function of different thresholds. However, thresholds were manual settings for triggering relocation requests and were not involved in optimization variables in that study.

Cepolina et al. (2012) [24] summarized existing studies intended to address the problem of vehicle relocation and categorized some of the approaches. Threshold based method was clearly organized. The authors deemed that the thresholds answer the question of where and when a relocation is required. The framework of thresholds was based on the proposal of Kek's paper. Cepolina et al. (2014) [25] applied thresholds to help planning a new urban carsharing system with fully automated personal vehicles, where they involved thresholds as a dependent variable in their model. The solution to this model is programmed as a parallel heuristic approach, which outputs the thresholds as a side product. Note that specific study on thresholds has not been conducted yet. Cepolina et al. (2015) [26] further developed three innovative strategies for urban carsharing systems where two of them were derived from thresholds method. They refer to article [25] when explaining the setting of thresholds. This confirms that the threshold method has potential for being developed for different application scenarios, and it would be valuable to provide further study on threshold determination.

Our previous work (2016) had presented a framework of threshold based relocation [27], which is based on the operating practice of EVCARD. We found that optimization methodology can hardly implement and handle operating in large scale networks and with stochastic demands, and threshold based relocation is fast practical and rather flexible to account for complex field circumstances. We model and determine the values of thresholds by a deterministic approach in the previous work. However, the randomness of demands and the property of electric vehicle are ignored at that time.

The purpose of this paper is to present a quantitative justification on thresholds determination. Moreover, the uncertainty of demands and trade-off between users' and operators' benefits are as well considered in this research.

\section{Thresholds Determination Modeling}

3.1. Problem Description. In each station, the number of vehicles is dynamically changing because vehicles would be picked up, dropped off, relocated in, and relocated out. For a single station, the changing range of vehicle amount depends on its own pick-ups and drop-offs, and its proper range is controlled by relocations.

As for the coherence with previous relocation-related works $[7,18]$, in this paper, time series is also discretized into time steps of 1 hour. During a time step, the vehicles dropped off, the vehicles relocated in, and the idle vehicles are all available for picking up and relocating out according to the matching between available state of charge (SOC) of these vehicles and the required travel distance.

If the number of vehicles at the station is below the lower threshold at one time step, the station requires vehicles to be relocated in. However, not all the required vehicles can be successfully moved into the station within this time step, and the exact arrivals of vehicles relocated in will endure a timestepwise delay. For the number exceeding the upper threshold it is similar, and the exact departure of vehicles to be relocated out will also delay due to the availability of relocation staff. 
Users want to select the vehicle whose battery life is the most suitable for their journey, and users will abandon the system at a proportion if no suitable vehicles are available. Vehicles will be charged immediately when arriving at station. Vehicles variation and depreciation are not concerned; battery charging is linear over time approximately; the battery status is denoted by the available travel distance, $\mathrm{km}$.

By modeling the dynamics of the number of vehicles at a station, the proper upper and lower thresholds can be determined under the minimization of the number of relocations and the time ratio where either the vehicles or parking spaces are out of service.

\subsection{State Transition}

3.2.1. Transition of the Number of Vehicles. The number of vehicles at the station in time step $t$ is formulated as

$$
x_{t}=x_{t-1}+r_{t}+\delta_{t}^{(+)}-d_{t}^{(s t)}-\delta_{t}^{(-)}, \quad \forall t=1,2, \cdots, T
$$

3.2.2. Vehicles to Be Moved In. The number of vehicles required to be moved in at $t$ is

$$
\delta_{t}^{(n+)}=\max \left(\left(S_{\text {lower }}-x_{t-1}\right), 0\right)
$$

For each vehicle $v_{t}=1,2, \cdots, \delta_{t}^{(n+)}$ that required to be moved in at $t$ and actually moved in at $t+t_{v_{t}}^{(+)}$, the moving tags and the sum of the moving tags of all $v_{t} s$ should be

$$
\begin{gathered}
v m_{t, t+t_{v_{t}}^{(+)}}^{(+), v_{t}}=1 \\
\sum_{v_{t}=1}^{\delta_{t}^{(n+)} v m_{t, t+t_{v_{t}}^{(+)}}^{(+), v_{t}}}=\delta_{t}^{(n+)}
\end{gathered}
$$

Each vehicle $v_{t} \in V_{t}^{(n+)}$ should be only moved once, from $t$ to the maximum delay time step:

$$
\sum_{v_{t}=1}^{\delta_{t}^{(n+)}} \sum_{t d=0}^{\max \left(t_{v_{t}}^{(+)}\right)} v m_{t, t+t d}^{(+), v_{t}}=\delta_{t}^{(n+)}
$$

The vehicles that actually moved in at $t$ :

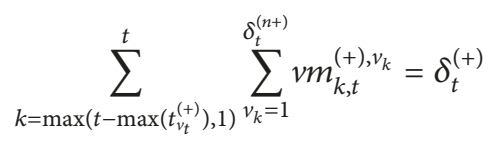

3.2.3. Vehicles to Be Moved Out. The number of vehicles required to be moved out at $t$ is

$$
\delta_{t}^{(n-)}=\max \left(\left(x_{t-1}-S_{u p p e r}\right), 0\right)
$$

For each vehicle $v_{t}=1,2, \cdots, \delta_{t}^{(n-)}$ that required to be moved out at $t$ and actually moved out at $t+t_{v_{t}}^{(-)}$, the moving tags and the sum of the moving tags of all $v_{t}$ s should equal $\delta_{t}^{(n-)}$ :

$$
\begin{gathered}
v m_{t, t+t_{v_{t}}^{(-)}}^{(-), v_{t}}=1 \\
\sum_{v_{t}=1}^{(n-)} v m_{t, t+t_{v_{t}}^{(-)}}^{(-), v_{t}}=\delta_{t}^{(n-)}
\end{gathered}
$$

Each vehicle $v_{t} \in V_{t}^{(n-)}$ should be only moved once, from $t$ to the maximum delay time step:

$$
\sum_{v_{t}=1}^{\delta_{t}^{(n-)}} \sum_{t d=0}^{\max \left(t_{v_{t}}^{(-)}\right)} v m_{t, t+t d}^{(-), v_{t}}=\delta_{t}^{(n-)}
$$

The vehicles that should be actually moved out at $t$ :

$$
\sum_{k=\max \left(t-\max \left(t_{v_{t}}^{(-)}\right), 1\right)}^{t} \sum_{v_{k}=1}^{\delta_{t}^{(n-)}} v m_{k, t}^{(-), v_{k}}=\delta_{t}^{*(-)}
$$

3.2.4. Vehicles Matching according to Battery Status. Denote $B_{t}=B_{t-1}^{V(r m)} \cup B_{t}^{(+)} \cup B_{t}^{(R)}$ and $B_{t}^{(D)}=B_{t-1}^{D(r m)} \cup B_{t}^{(g e)}$. Supposing demands of pick-ups and requests to relocate vehicles out have equal opportunity to select vehicles, the matching processing should satisfy the following conditions.

For each available vehicle, there should be at most one demand matching it:

$$
\sum_{b^{(D)} \in B_{t}^{(-)} \cup B_{t}^{(D)}} v s_{t}^{b, b^{(D)}} \leq 1, \quad \forall b \in B_{t}
$$

For each demand, there should be at most one available vehicle matching it:

$$
\sum_{b \in B_{t}} v s_{t}^{b, b^{(D)}} \leq 1, \quad \forall b^{(D)} \in B_{t}^{(-)} \cup B_{t}^{(D)}
$$

For available vehicles and demands matching, we suppose a "best matching" principle that users and relocation staff will get the vehicles with the best utility, leading to a maximum of the total provided and demanded battery of matched vehicles:

$$
\begin{aligned}
& \sum_{b \in B_{t}} \sum_{b^{(D)} \in B_{t}^{(-)} \cup B_{t}^{(D)}} b \times b^{(D)} \times v s_{t}^{b, b^{(D)}} \\
&-\sum_{b \in B_{t}} \sum_{b^{(D)} \in B_{t}^{(-)} \cup B_{t}^{(D)}} b \times b^{(D)} \times \overline{v s}_{t}^{b, b^{(D)}} \geq 0, \\
& \forall \overline{v s}_{t}^{b, b^{(D)}} \in V S
\end{aligned}
$$

where $V S$ denotes a space containing all combinations of $v s_{t}^{b, b^{(D)}}$.

It should be noted that the matching between the demands and the available vehicles according to the desired and the provided battery status is actually even complicated, and further studies on this problem can be derived.

Hence, the actual number of vehicles to be relocated out at $t$ should be

$$
\delta_{t}^{(-)}=\sum_{b \in B_{t}} \sum_{b^{(D)} \in B_{t}^{(-)}} v s_{t}^{b, b^{(D)}}
$$


The actual number of satisfied demands at $t$ should be

$$
d_{t}^{(s t)}=\sum_{b \in B_{t}} \sum_{b^{(D)} \in B_{t}^{(D)}} v s_{t}^{b, b^{(D)}}
$$

The battery status of the remaining vehicles at the end of time step $t$ consists of a set:

$$
\begin{gathered}
B_{t}^{V(r m)}=\left\{\min \left(b+l \cdot c v, B_{\text {max }}\right) \mid \forall b\right. \\
\left.\in B_{t}, \quad \sum_{b^{(D)} \in B_{t}^{(-)} \cup B_{t}^{(D)}} v s_{t}^{b, b^{(D)}}=0\right\}
\end{gathered}
$$

The unsatisfied demand battery status set is

$$
B_{t}^{D(u s)}=\left\{b \mid \forall b \in B_{t}, \quad \sum_{b^{(D)} \in B_{t}^{(-)} \cup B_{t}^{(D)}} v s_{t}^{b, b^{(D)}}=0\right\}
$$

The remaining demand battery status set is

$$
B_{t}^{D(r m)}=\left\{b \mid \forall b \in B_{t}^{D(u s)}, p_{t}^{b}=1\right\}
$$

3.3. Thresholds Determination. The optimization model of thresholds determination is formulated as follows.

Objective 1 (CSOs' perspective)

$$
\begin{aligned}
& \min f_{1}\left(S_{\text {upper }}, S_{\text {lower }}\right) \\
& =-\left(R V \cdot \sum_{t=1}^{T} d_{t}^{(s t)}-C R \cdot \sum_{t=1}^{T} \delta_{t}^{(-)}\right)
\end{aligned}
$$

Objective 2 (users' perspective)

$$
\begin{array}{ll}
\min & f_{2}\left(S_{\text {upper }}, S_{\text {lower }}\right)=\frac{\left(\sum_{t=1}^{T} u_{t}^{(+)}+\sum_{t=1}^{T} u_{t}^{(-)}\right)}{T} \\
\text { s.t. } & S_{\text {lower }} \leq S_{\text {upper }} \\
& S_{\text {upper }} \leq N \\
& S_{\text {lower }} \geq 0 \\
& S_{\text {lower }}, S_{\text {upper }} \text { are integers, }
\end{array}
$$

with state transition equations (1) to (19).

Objective 1 indicates that the CSOs' objective is to maximize the profits at the station. Formula (20) is to add up the revenues from all satisfied demands and subtract the costs of all the relocations. Formula (21) describes the ratio of the time that the system is unavailable for both pick-up and dropoff requirement to the total time, which indicates the service quality of the system. The decision variables $S_{\text {upper }}, S_{\text {lower }}$ are hidden in both objective functions. Formulae (22) to (25) constrain the thresholds where the lower threshold should be equal to or lower than the upper threshold, the upper threshold should not exceed the number of parking space, and the lower threshold should not be below 0 . The state transition equations are also constraints to guarantee the dynamics of the system.

\section{Solution Algorithm}

4.1. Solution Principles. The thresholds determination model under stochastic demands is facing three problems:

(a) The stochastic variables make the other parameters and variables uncertain during the state transition and let the definition of the minimization of the objectives be uncertain.

(b) The two objectives are conflicting with each other and hard to achieve at the same time, which is not explicit for decision, and compromises need to be made.

(c) The model is hard to be linearized due to the hidden property of decision variables, the dynamic change of system parameters, and the uncertainty of the stochastic variables and sets.

To solve this model, reformations are given as follows.

For problem (a), employ the min-max robust optimization to find out the best solutions under the worst conditions of the stochastic variables, and rewrite the objective as

$$
\begin{aligned}
& \min _{S_{\text {upper }}, S_{\text {lower }}}\left\{\max _{\substack{r_{t}, d_{t}^{(g e)}, b_{t, v_{t}}^{(R)}, b_{t, v_{t}}^{(g e)}, b_{t, v_{t}}^{(+)}, b_{t, v_{t}}^{(-)}, t_{v_{t}}^{(+)}, t_{v_{t}}^{(-)}, p_{t}^{b}}} f_{i}\left(S_{\text {upper }}, S_{\text {lower }}\right), \forall t\right. \\
& =1,2, \cdots, T, \forall i=1,2\}, \quad \forall i=1,2
\end{aligned}
$$

In objective (26), the inner objective is to locate the maximum (worst) metrics and find out the values of the stochastic variables (auxiliary decision variables) under the worst conditions; the outer objective is to judge the minimum (best) metrics among worst conditions of all the $S_{\text {upper }} \mathrm{s}$ and $S_{\text {lower }} \mathrm{s}$ and decide the final solutions on $S_{\text {upper }}$ and $S_{\text {lower }}$. This strengthens the robustness of the solutions, i.e., ensures the result thresholds to be capable during the worst condition under the stochastic demands.

Problem (b) can be addressed by introducing the Pareto optimum, which determines a set of nondominated solutions where improvement of one objective cannot be achieved without sacrifice of the other objective, and the objective metrics of these solutions lie on the Pareto front. To define the Pareto solution set, a decision vector $\vec{x}^{*}$ is Pareto optimal solution if there does not exist another $\vec{x}$ such that $f_{i}(\vec{x}) \leq$ $f_{i}\left(\vec{x}^{*}\right), \forall i=1,2$ and $f_{j}(\vec{x})<f_{j}\left(\vec{x}^{*}\right)$ for at least one $j$. For both "min" and "max" steps the Pareto optimum 
creates rational solution sets and Pareto fronts to pick out compromised solutions under both objectives.

To construct the solution algorithm and cope with the problem (c), we involve the Monte Carlo approach to generate the stochastic seeds and determine the final solutions according to the previous rules. Detailed solution procedures are illustrated in Section 4.2.

4.2. Solution Procedure. In the study case of EVCARD, because the maximum $N$ is 10 among all stations, the combinations of $S_{\text {upper }}$ and $S_{\text {lower }}$ can be enumerated and the random seeds can be generated through Monte Carlo approach, so the enumerative solution procedure is proposed as follows.

Step 1. For each $S_{\text {upper }}$ and $S_{\text {lower }}$ satisfying the constraints, create pairs of $S_{\text {upper }}$ and $S_{\text {lower }}$; for each pair of thresholds we have the following.

Step 1.1. For stochastic variables $\left\{r_{t}, d_{t}^{(g e)}, b_{t, v_{t}}^{(R)}, b_{t, v_{t}}^{(g e)}, b_{t, v_{t}}^{(+)}\right.$, $\left.b_{t, v_{t}}^{(-)}, t_{v_{t}}^{(+)}, t_{v_{t}}^{(-)}, p_{t}^{b}\right\}$, which should follow their own distribution, generate $R$ random seeds.

Step 1.2. For each random seed, calculate the two objectives through the state transition equations, denote these solutions as $k_{r}, \forall r=1, \cdots, R$, with decision variables $S_{\text {upper }}^{k_{r}}, S_{\text {lower }}^{k_{r}}$ and objectives $f_{1}^{k_{r}}, f_{2}^{k_{r}}$; add $k_{r}, \forall r=1, \cdots, R$ to a set $K_{a}$ which includes all stochastic solutions.

Step 1.3. From the calculated objectives corresponding to the random seeds, find out the worst-case Pareto front of this pair of $S_{\text {upper }}$ and $S_{\text {lower }}$; add these solutions to a set denoted as $K_{S_{\text {upper }}, S_{\text {lower }}}^{w}$.

Step 1.4. If every pair of $S_{\text {upper }}$ and $S_{\text {lower }}$ has been processed, continue to Step 2.

Step 2. For all pairs of $S_{\text {upper }}$ and $S_{\text {lower }}$, find out the bestcase Pareto front in set $\bigcup_{S_{\text {upper }}, S_{\text {lower }}} K_{S_{\text {upper }}, S_{\text {lower }}}^{w}$, as well as the corresponding nondominated solutions; add these solutions to a set denoted as $K$.

Step 3. Pick out final decision. For solution $k \in K$ with decision variables $S_{\text {upper }}^{k}, S_{\text {lower }}^{k}$ and objectives $f_{1}^{k}, f_{2}^{k}$, since $f_{1}^{k} \in[-\infty,+\infty)$ and $f_{2}^{k} \in[0,1]$, to judge both objectives fairly, normalize the two objectives as

$$
\begin{aligned}
& f n_{1}^{k} \\
& =\frac{\left(\left(f_{1}^{k}-\min _{m \in K_{a}} f_{1}^{m}\right)-\min _{m^{\prime} \in K_{a}}\left(f_{1}^{m^{\prime}}-\min _{m \in K_{a}} f_{1}^{m}\right)\right)}{\max _{m^{\prime} \in K_{a}}\left(f_{1}^{m^{\prime}}-\min _{m \in K_{a}} f_{1}^{m}\right)} \\
& f n_{2}^{k}=\frac{\left(f_{2}^{k}-\min _{m \in K_{a}} f_{2}^{m}\right)}{\max _{m \in K_{a}} f_{2}^{m}}
\end{aligned}
$$

Define the weighted objective as

$$
f^{k}=\omega_{1} f n_{1}^{k}+\omega_{2} f n_{1}^{k}
$$

where $\omega_{1}$ and $\omega_{2}$ are weights and $\omega_{1}+\omega_{2}=1$, which represent the importance of each objective and could be set by the operators. For the operators that attach more importance to objective 1 over objective $2, \omega_{1}$ could be set higher than $\omega_{2}$. Find out $k^{*} \in K$ where $f^{k^{*}}=\min _{k \in K} f^{k}$, and $k^{*}$ is the final decision, where the thresholds are $S_{\text {upper }}^{k^{*}}, S_{\text {lower }}^{k^{*}}$, with the objectives of $f_{1}^{k^{*}}, f_{2}^{k^{*}}$.

\section{Analysis and Discussion}

5.1. Calibration of Stochastic Distribution. The data bed to calibrate the stochastic variables is based on the order record database of EVCARD during April 2015, which contained 2848 order records as well as the information of 56 stations, and the supplementary user survey conducted in March 2016, which retrieved 138 respondents' intention.

(1) Demand variables $r_{t}$ and $d_{t}^{(g e)}$ : One day is discretized into 24 time periods by the step width $1 \mathrm{~h}$, and each period over a month is independent and follows the same distribution. Poisson distributions are tested for them. Figure 2 displays the comparison between the real data histograms (displayed in blue bars) and the theoretical Poisson distributions (displayed in orange bars) at station No. 44 Jiading campus among all the time steps in one day. Their distribution hypothesis tests are conducted by $\chi^{2}$-test under significance level 0.05 , where $h=0$ denotes not rejecting the null hypothesis of Poisson and $\mathrm{h}=1$ means reject.

(2) Continuous variables $b_{t, v_{t}}^{(R)}, b_{t, v_{t}}^{(g e)}, b_{t, v_{t}}^{(+)}, b_{t, v_{t}}^{(-)}$: The battery status follows continuous distribution. We investigated the battery status of returned vehicles and relocated vehicles through the orders and the users' desired battery status by the user survey. The histograms and the hypothesized distribution are illustrated in Figure 3. Under significance level 0.05 , K-S tests were conducted to exam the fitness of continuous distribution and all of the four distributions ((a) uniform, (b) log-normal, (c) log-normal, and (d) normal) are not rejected.

(3) Discretized variables $t_{v_{t}}^{(+)}, t_{v_{t}}^{(-)}, p_{t}^{b}$ : The relocation time delay is inferred from the order data and the demands remaining probability is inferred from the user survey. Figure 3 reveals the distribution of the discretized time delay and rejected demands remaining. Figure 3(e) (or Figure 3(f)) shows the probability of delay for 0 time step, 1 time step, 2 time steps, etc. Figure 3(g) refers to the abandon proportion (denoted by 0 ) and the remaining proportion (denoted by 1 ) when the demands are rejected due to the vehicle insufficiency as a 0-1 distribution, where more than a half users prefer to abandon using the system.

5.2. Numerical Example. The data of the example came from the station No. 44, Jiading Campus, of the EVCARD system during Apr. 2015. There are seven parking spaces $(N=7)$ at the station; the time step width $l=1 \mathrm{~h}$; for 30 days' data there are 720 time steps during the study period $(T=720)$. The general vehicle type can travel at most $100 \mathrm{~km}$ and will be charged in $5 \mathrm{~h}$ in AC charging mode $\left(c v=20, B_{\text {max }}=100\right)$.

Figure 4 shows the generation of the random seeds by the scatters of the two objectives. In the figure, different colors 


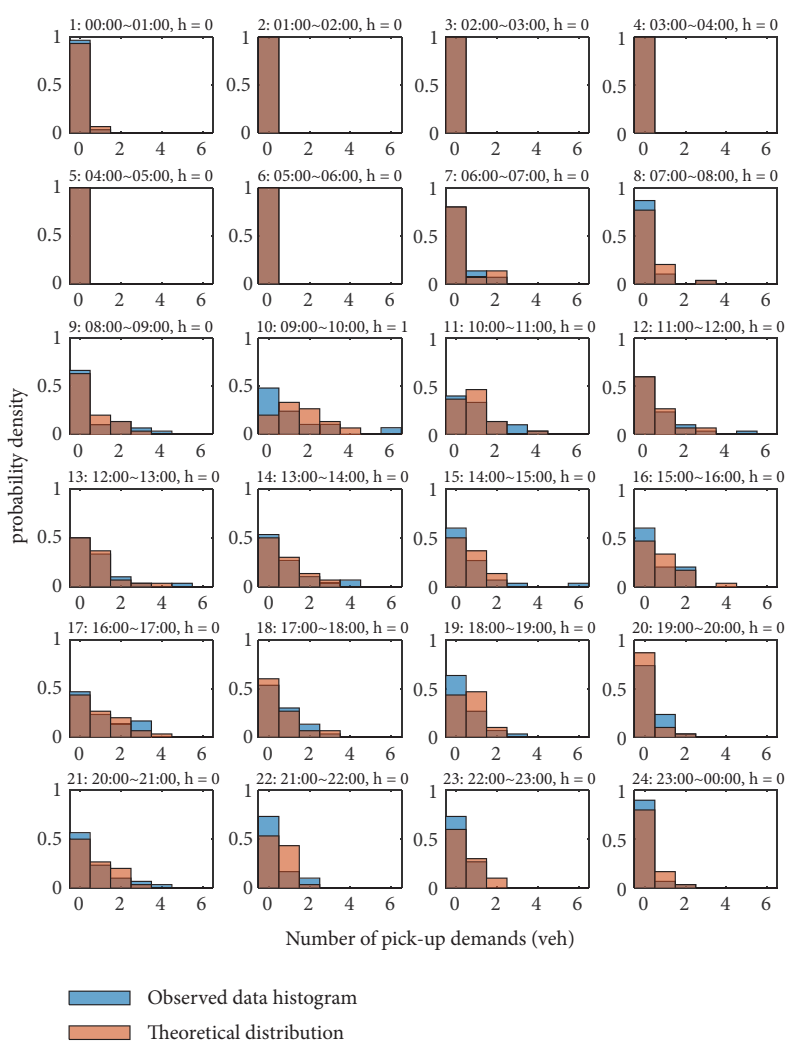

(a) Pick-up distribution

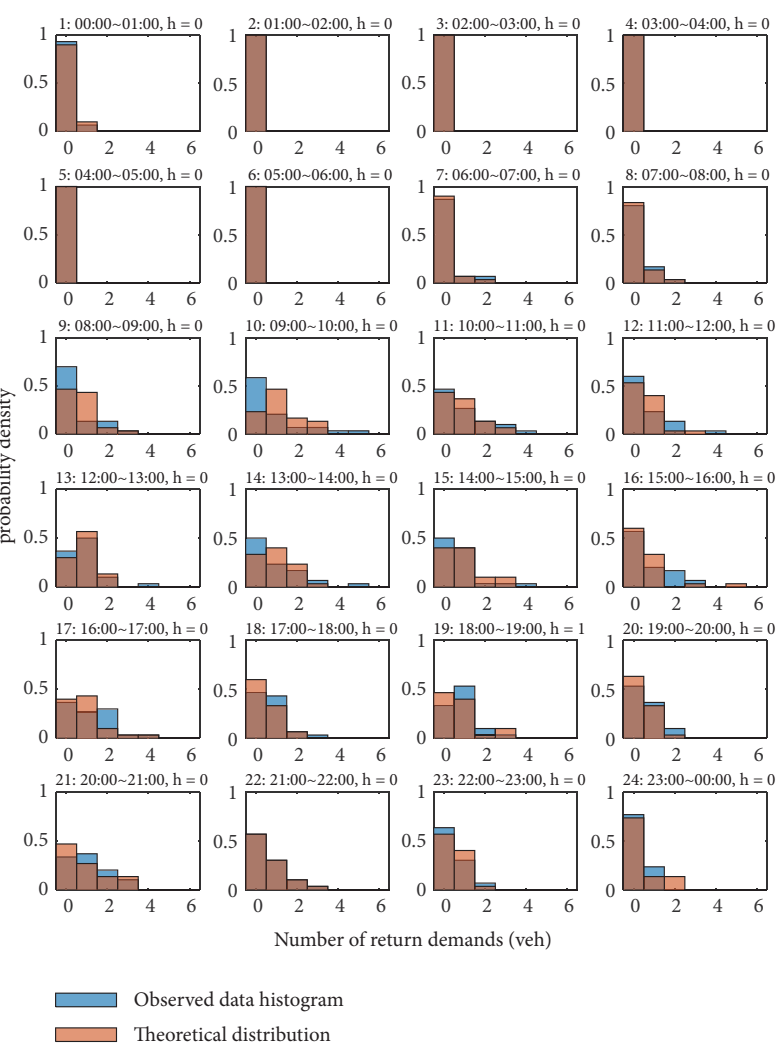

(b) Return distribution

Figure 2: Observed histogram and theoretical distribution of pick-up and return demands.

are used to distinguish different pairs of $S_{\text {upper }}$ and $S_{\text {lower }}$; dots denote the objective values of all the random seeds, stars denote the worst-case Pareto front of each pair of $S_{\text {upper }}$ and $S_{\text {lower }}$, and stars with circles denote the minimum Pareto front in all the worst conditions. Step 1 of the algorithm can be visualized in Figure 4(a) where the random seeds are generated for each pair of thresholds and the worst Pareto fronts of each pair of thresholds are highlighted in stars. Step 2 is visualized in Figure 4(b) which retained the worst-case Pareto fronts and the best-case Pareto front is approximated based on the worst-case Pareto fronts and marked by circles.

The nondominated solutions of thresholds and corresponding minimum Pareto front are listed in Table 1. Thresholds $(3,4),(2,5),(3,5),(0,6),(1,6)$, and $(2,6)$ are all reasonable to guarantee the worst condition caused by the random demands. To decide the final decision for CSOs according to Step 3 of the algorithm, we need to select values of weights. Generally, operators attach equal importance to both of the objectives, like the case of EVCARD in this study. We attached equal importance to both objectives in previous study [27] through deterministic approach (weight $\omega_{1}=\omega_{2}$ ). For comparison, we also choose equal weights here. Under this metric, $(2,6)$ will be selected as the final decision as the thresholds of the station.

5.3. Comparison among Determination Methods. Three means of thresholds determination methods are compared. (1) Empirical method: The upper thresholds are set by the number of parking space minus 1 to satisfy the drop-offs, and the lower thresholds are set as 1 to ensure a vehicle available. (2) Deterministic method: The random variables are substituted by the known order data, and optimal solutions of thresholds are calculated aiming to minimize the relocation times as well as the out-of-service rate. (3) Stochastic method is proposed by this paper.

Based on the same data bed, we calculated the thresholds of all the 56 stations using the three methods. Figure 5 illustrates a comparison among the results of the three methods. The results of the 56 stations using the three methods appear with both differences and indiscrimination, which are concluded in Table 2.

Notably, there are 27 in 56 stations where the three methods give all different solutions and six stations where the stochastic method gives different solutions from empirical method and deterministic method (as shown in Table 3), which means there are 33 stations (59\%) where the stochastic method reaches other solutions. However, for 13 stations (23\%) the stochastic method solves out the same solutions as the empirical rules do, and for 7 stations the three methods come to the same results; i.e., empirical method gives identical answer as the stochastic method does in 20 stations (36\%). The deterministic method only gives identical results to the stochastic methods in 13 stations (23\%).

Except the 7 stations where the three methods give the same solutions, we extract four representative stations under uncertain demands, respectively, from the condition that 


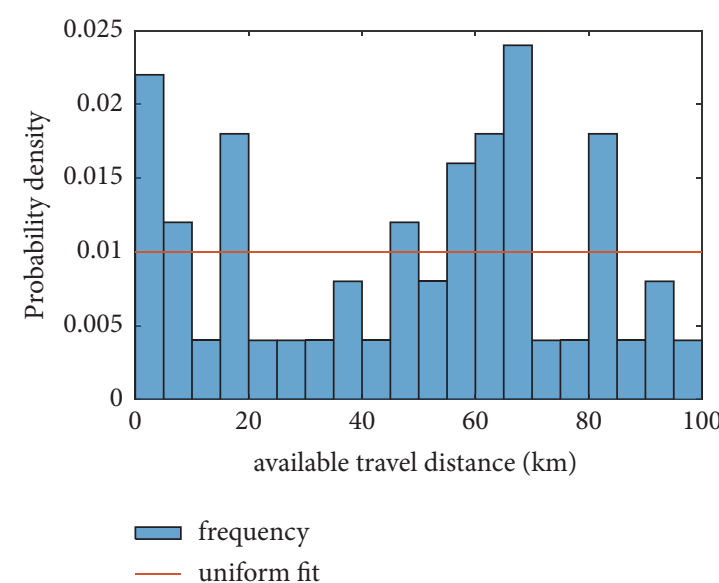

(a) Distribution of battery status of returned vehicles

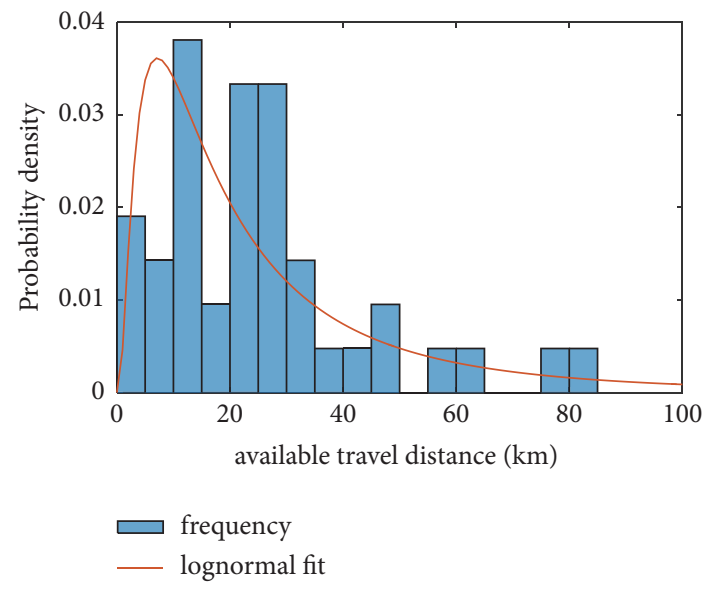

(c) Distribution of battery status of relocate-in

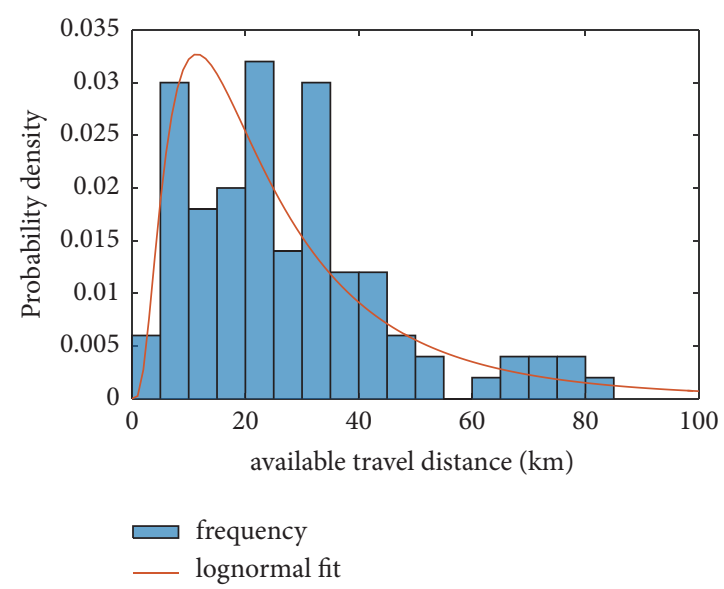

(b) Distribution of users' desired battery status

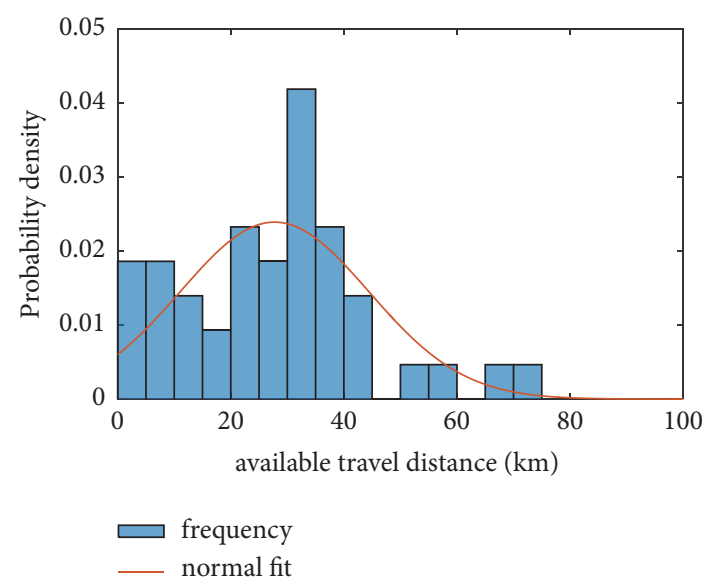

(d) Distribution of battery status of relocate-out

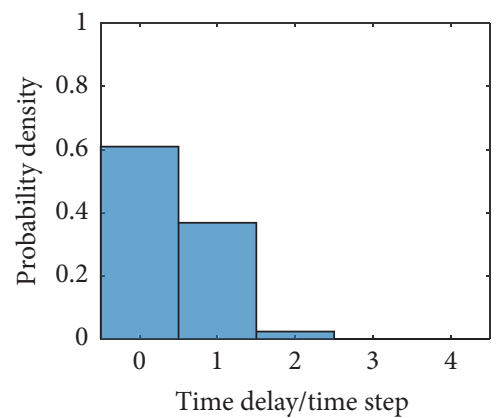

(e) Relocate-in time delay

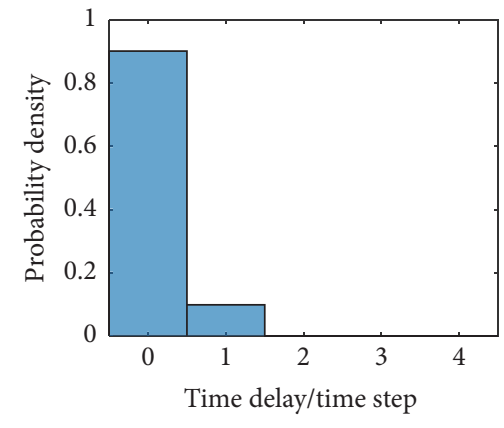

(f) Relocate-out time delay

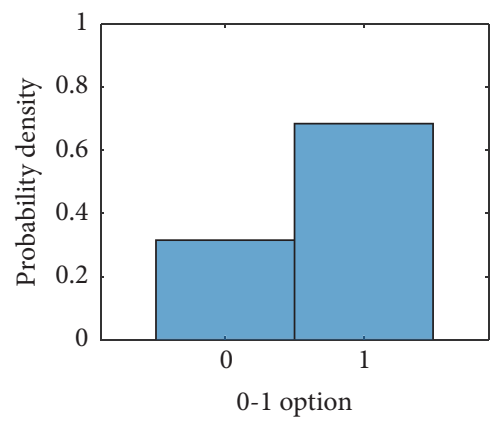

(g) Demands remaining

FIGURE 3: Distribution of discretized variables.

the empirical method equals the deterministic method, the condition that the empirical method equals the stochastic method, the condition that the deterministic method equals the stochastic method, and the condition that they are all different, and the scatterplots and histograms of both objective metrics of four stations are shown in Figures 6(a), 6(b), 6(c), and 6(d), respectively. In Figure 6, random seeds are denoted by dots, the worst-case Pareto fronts are denoted by color circles and solid links, and the best-case Pareto fronts are denoted by black circles and dash links. For station No. 36 in Figure 6(a) the solution from stochastic method performs better than the solution from the empirical method and deterministic method in out-of-service rate with minor concession on profits. For station No. 34 in Figure 6(b), the stochastic method as well as empirical rules perform obviously better on out-of-service rate. For station No. 29 in Figure 6(c), the stochastic method and the deterministic method achieve absolutely lower out-of-service rate with 
TABLE 2: The nondominated solutions set and the final decision under weighted method.

\begin{tabular}{lcccc}
\hline Lower threshold & Upper threshold & Profits of the operator & out of service Rate & Weighted objective \\
$\boldsymbol{S}_{\text {lower }}$ & $\boldsymbol{S}_{\text {upper }}$ & $\boldsymbol{f}_{\mathbf{1}}$ & $\boldsymbol{f}_{\mathbf{2}}$ & $\boldsymbol{f}$ \\
\hline 3 & 4 & -2407 & 0.001 & 0.500 \\
2 & 5 & -4343 & 0.004 & 0.260 \\
3 & 5 & -5023 & 0.010 & 0.187 \\
0 & 6 & -6230 & 0.193 & 0.471 \\
0 & 6 & -6339 & 0.210 & 0.497 \\
1 & 6 & -6113 & 0.054 & 0.155 \\
$\mathbf{2}$ & $\mathbf{6}$ & -5851 & $\mathbf{0 . 0 2 8}$ & $\mathbf{0 . 1 2 5}$ \\
\hline
\end{tabular}

TABLE 3: Differences and indiscrimination of results from the three methods.

\begin{tabular}{lccccc}
\hline & All the same & Emp eq. Det & Emp eq. Sto & Det eq. Sto & All different \\
\hline The number of stations & 7 & 6 & 13 & 3 & 27 \\
\hline
\end{tabular}

Note: Emp denotes the empirical method; Det denotes the deterministic method; Sto denotes the stochastic method; eq. denotes equals.

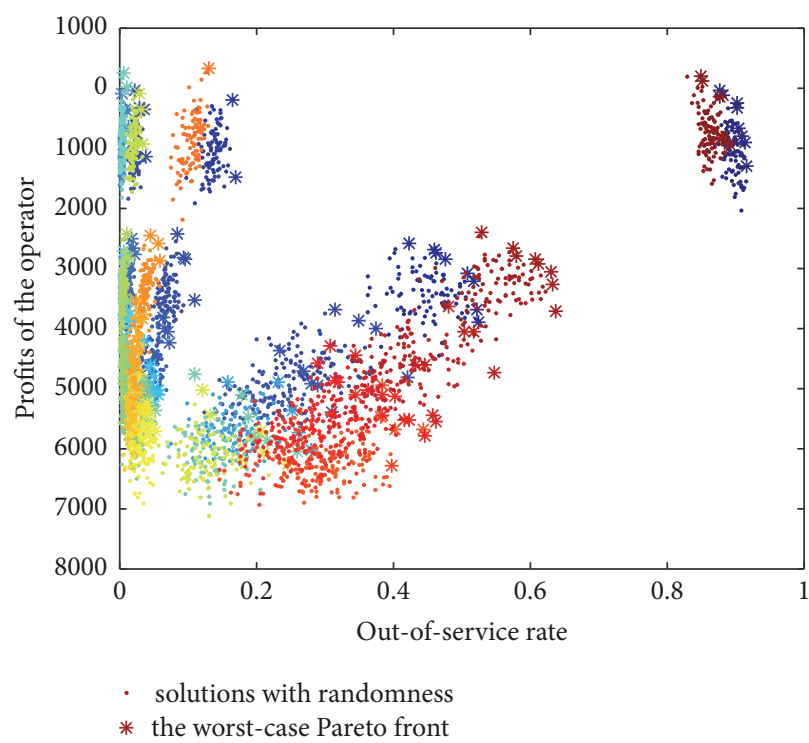

(a) Determination of the worst-case Pareto front

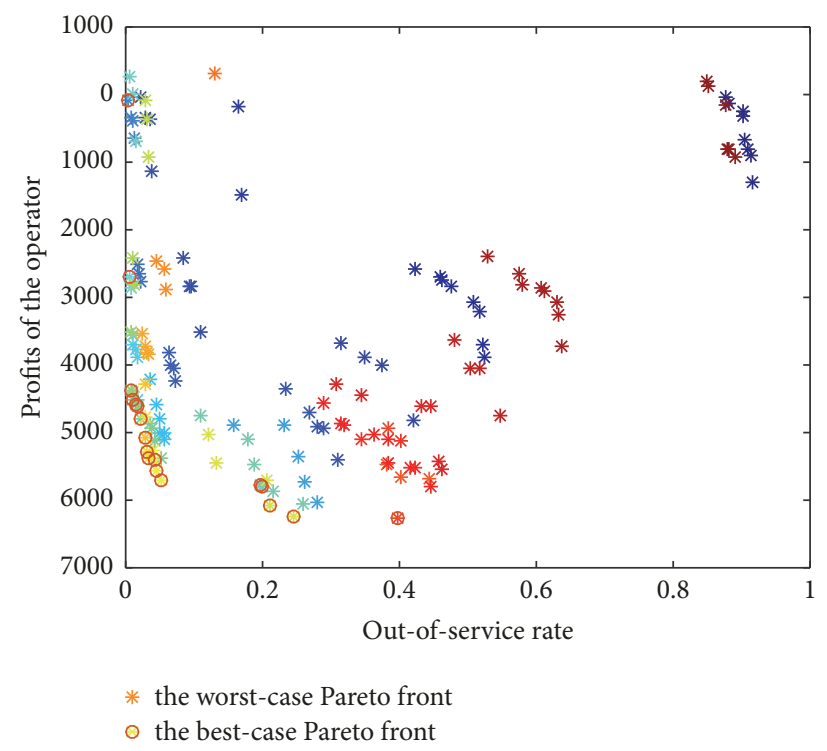

(b) Determination of the best-case Pareto front

FIgURE 4: The visualization of the algorithm of the example station.

approximate profits. For station No. 44 in Figure 6(d) the worst condition of stochastic method performs better than the worst condition of other methods, with little sacrificing on profits.

5.4. Variable Thresholds during a Day. The above model captures long term characteristics and determined a daily thresholds scheme, which supports day-to-day operation. However, for more specific situation, demands can appear with variation in different period of day. To account for variations depending on time-of-day, we specify the daily thresholds into variable thresholds during a day.
The decision variables should be extended to all time steps, and $\forall t=1,2, \cdots, T$, we have the following decision variables:

$$
\begin{aligned}
& S_{\text {upper }}^{t} \text { : upper threshold of the station at time step } t \\
& S_{\text {lower }}^{t} \text { : lower threshold of the station at time step } t .
\end{aligned}
$$

And the formulae in state transition functions (2) and (7) should be modified as

$$
\begin{aligned}
& \delta_{t}^{(n+)}=\max \left(\left(S_{\text {lower }}^{t}-x_{t-1}\right), 0\right) \\
& \delta_{t}^{(n-)}=\max \left(\left(x_{t-1}-S_{\text {upper }}^{t}\right), 0\right)
\end{aligned}
$$




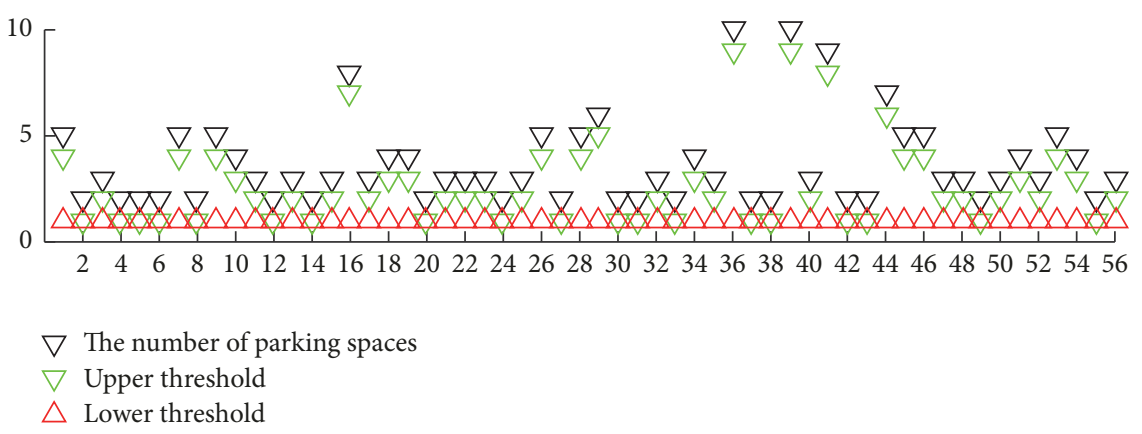

(a) Thresholds determined by empirical method

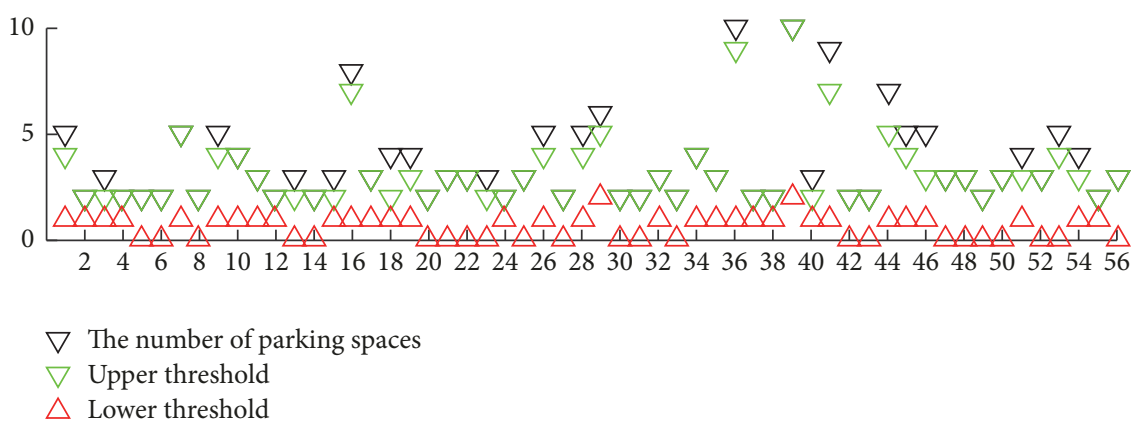

(b) Thresholds determined by deterministic method

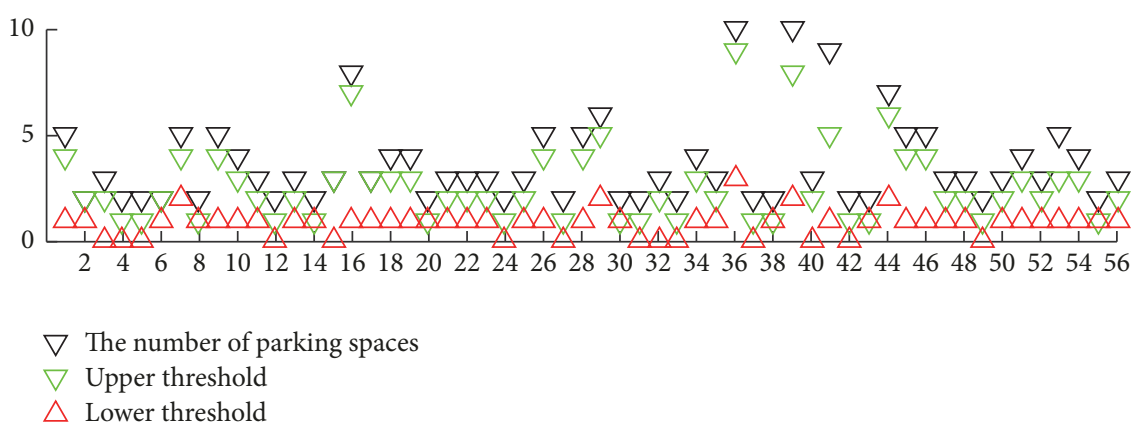

(c) Thresholds determined by stochastic method

FIGURE 5: Result comparison among empirical method, deterministic method, and stochastic method.

The constraints regarding upper and lower thresholds should also be

$$
\begin{gathered}
S_{\text {lower }}^{t} \leq S_{\text {upper }}^{t}, \\
S_{\text {upper }}^{t} \leq N, \\
S_{\text {lower }}^{t} \geq 0, \\
S_{\text {lower }}^{t}, S_{\text {upper }}^{t} \text { are integers, } \\
\forall t=1,2, \cdots, T
\end{gathered}
$$

The previous model can be fast solved due to the limited combination of two thresholds. In the extended model, more thresholds responding to different time steps significantly enlarge the space of the solutions. There are $N(N-1) / 2$ combinations of two thresholds but $(N(N-1) / 2)^{t}$ combinations of $t$ time steps. Supposing there are 24 periods in a day as the research time range, the solution space turns out to be quite huge.

Considering the dynamics of the system happens by time step successively, a local optimum can be reached from a dynamic programming approach. We solve one pair of thresholds for one time step from $t=1$ to $T$ step by step. Firstly $\left(S_{\text {lower }}^{1}, S_{\text {upper }}^{1}\right)$ can be solved. When solving $\left(S_{\text {lower }}^{t}, S_{\text {upper }}^{t}\right)$, we deem the previous feasible solution of $\left(S_{\text {lower }}^{1}, S_{\text {upper }}^{1}\right), \ldots,\left(S_{\text {lower }}^{t-1}, S_{\text {upper }}^{t-1}\right)$ as known variables solved in previous steps. In that case the algorithm in this paper is applicable to solve the variable thresholds problem.

Figure 7 shows the case to determine the thresholds of station No. 44. Figure 7(a) illustrates the demand pattern in different time steps, where boxplots indicate the randomness of demands. Figure 7(b) shows the result of $\left(S_{\text {lower }}^{t}, S_{\text {upper }}^{t}\right)$, $\forall t=1,2, \cdots, 24$, during one day, where green triangles denote the upper thresholds and red triangles denote the 

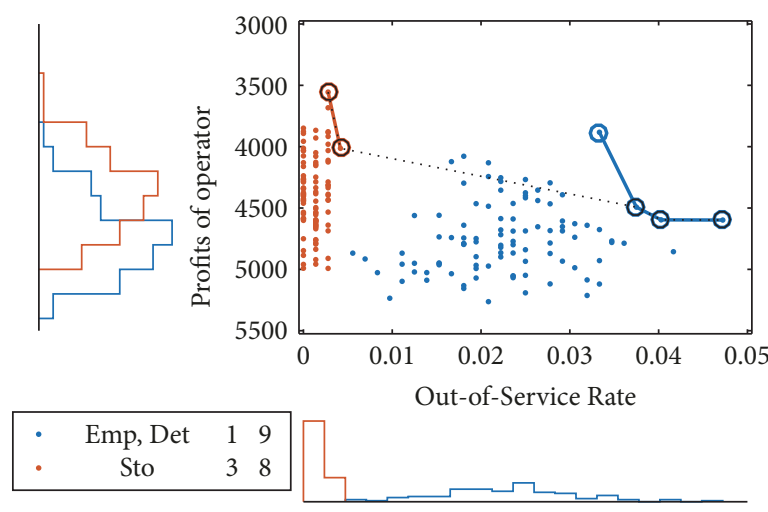

Emp: Empirical method $\bigcirc \bigcirc$ Worst-case Pareto front Det: Deterministic method $\quad \because \cdots \because$ Best-case Pareto front Sto: Stochastic method

(a) Station $36, N=10$
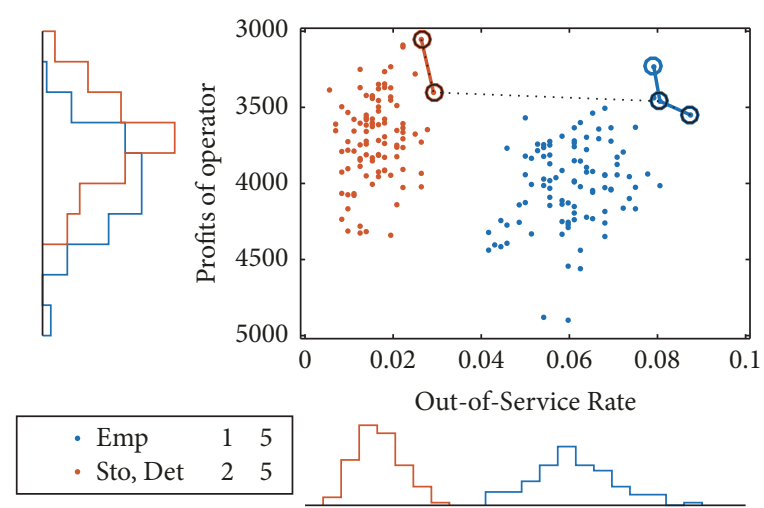

Emp: Empirical method $\quad \bigcirc \bigcirc$ Worst-case Pareto front
Det: Deterministic method
Sto: Stochastic method

(c) Station $29, N=6$
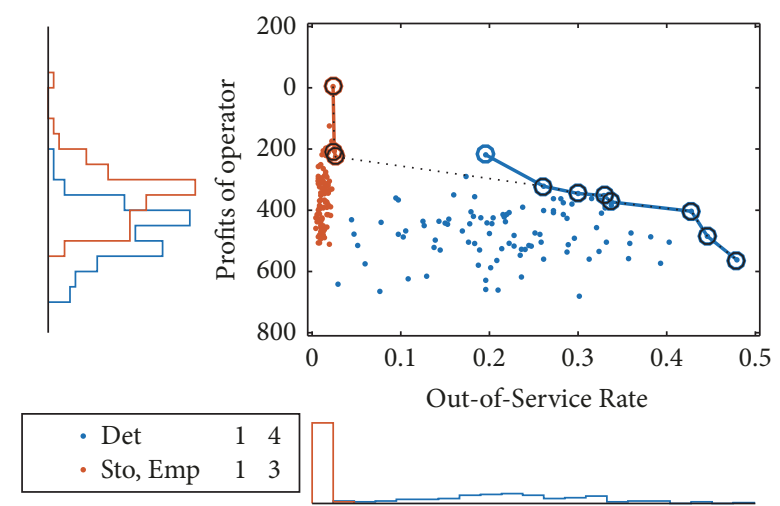

Emp: Empirical method $\bigcirc \bigcirc$ Worst-case Pareto front Det: Deterministic method $\quad \because \cdots \cdots$ Best-case Pareto front Sto: Stochastic method

(b) Station $34, N=4$
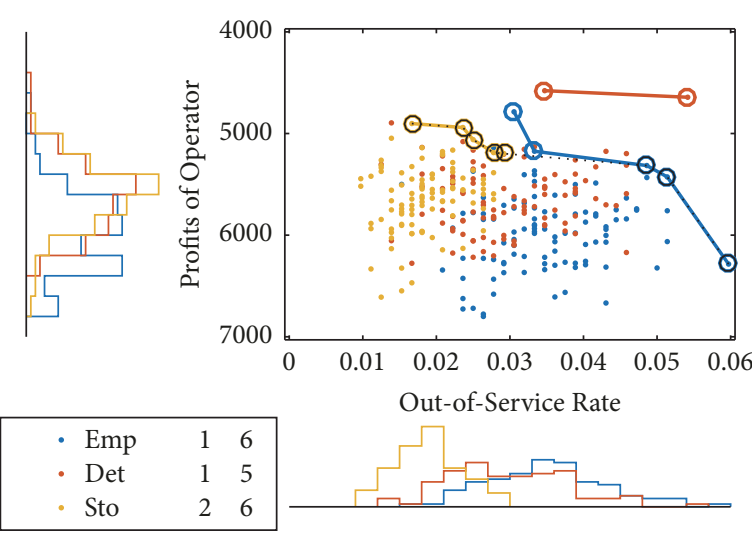

Emp: Empirical method $\bigcirc$ Worst-case Pareto front Det: Deterministic method $\quad \because \cdots \cdots$ Best-case Pareto front Sto: Stochastic method

(d) Station $44, N=7$

FIGURE 6: Comparison of the profit among the three methods.

lower thresholds. The solid line in this figure represents the average possible number of vehicles conserved in the station. The green and red dash line, respectively, indicate the possible maximum and minimum bound of vehicles conserved in the station due to the stochastic demands. The green and red dash lines confine a probable range of the numbers of vehicles at the station.

There are some findings from the result shown by the figure. During the wee hours $(1: 00 \sim 6: 00)$ with low demands, the upper threshold is relatively low, which allows idle vehicles to be moved out, to support other stations which need vehicles to be moved in during the same period (if existing). When demands occur after 7:00, the lower threshold goes up for requiring vehicles to be moved in, accounting for the following peak hours. Until 16:00 demands slightly decrease, and the possible range of vehicle number at station during 16:00 seems to be relatively higher, so that the thresholds accordingly go down to let the vehicles to be moved out.
During 18:00 the possible range of vehicle number seems to decrease and the thresholds allow vehicles to be moved in. After 20:00 the number of vehicles turns to be stable and the thresholds are wider to let the number of vehicles change naturally.

\section{Conclusions}

This paper presents an approach on determining the thresholds considering the stochastic demands and system states, which provides a quantitative justification for thresholds determination. Firstly, we establish a model to capture the dynamics of the system status involving stochasticity and an optimization tactic is employed to find out proper values of upper and lower thresholds, aiming at minimizing the relocation times and the out-of-service time rate. The solution algorithm is provided to determine the rational solutions for this model, employing the min-max robust optimization to 


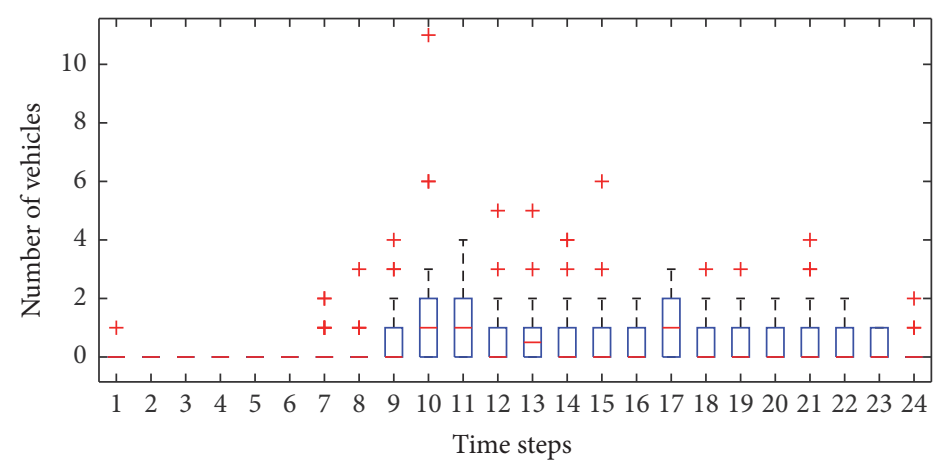

(a) Boxplot of stochastic demand pattern in different time steps

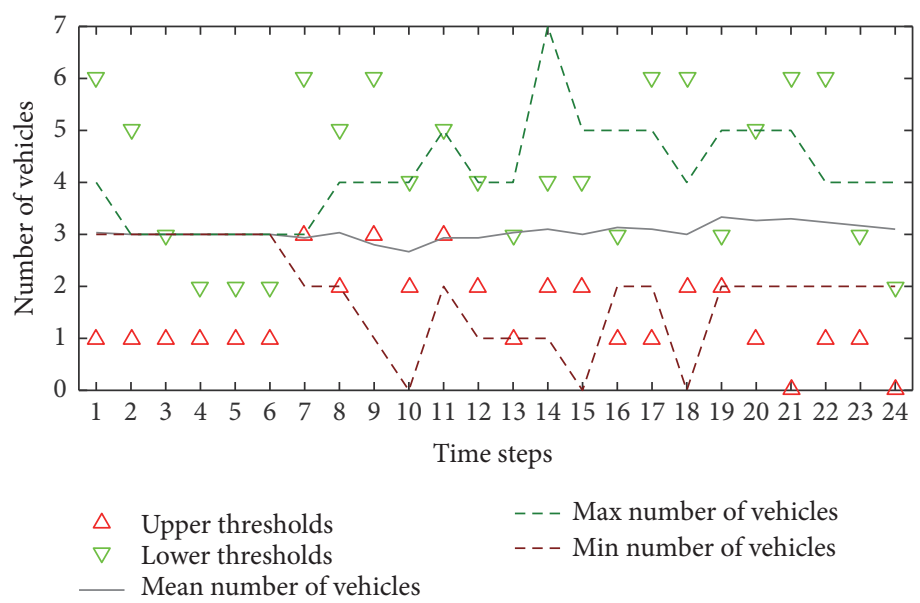

(b) Thresholds in different time steps

FIgURE 7: Solution of thresholds for different hours in a day.

tackle the uncertainty and the Pareto optimum to decide the solution under dual objectives.

To calibrate the stochastic variables, the continuous variables and the discretized variables are tested under the significance level of 0.05 , which did not reject their hypothesis on their distribution, respectively. A numerical example was given to demonstrate the algorithm. A comparison was conducted among the empirical rules, the deterministic method, and the stochastic method, where the results suggest that the stochastic rules get the identical results with the empirical method at $23 \%$ of the stations and deterministic method at 5\%. Among the stations where the stochastic method outputs different results from the empirical or deterministic method, the stochastic method achieves better solutions, which confirm that the stochastic method provides more reasonable justification towards uncertain demands.

Actually the threshold determination is a fundamental part of the threshold based relocation approach; the entire method considering stochastic demands and system status is not sufficiently studied. Although the threshold based method is an on-demand respond method, the efficiency and effectiveness of this method in practice have not been reported yet. In the future, dynamic control or operation approaches are promising to cope with the real time relocation problem, which will bring more profits to the carsharing system operation.

\section{Notations}

(a) Parameters

T: $\quad$ The number of time steps

$t: \quad$ Time step $t, t=0,1,2, \cdots, T$

$N$ : The number of parking spaces at the station

$l$ : $\quad$ Time step width, $\mathrm{h}$, in this paper $l=1$

$c v$ : Charging rate of the vehicle, $\mathrm{km} / \mathrm{h}$

$C R$ : Cost of relocation for each trip

$R V: \quad$ Average revenue of each trip departing from the station

$B_{\text {max }}$ : The maximum battery status, which is described as continuing travel distance, $\mathrm{km}$. 


\section{(b) Decision Variables}

\section{(1) Primary Decision Variables}

$S_{\text {upper }}$ : Upper threshold of the station

$S_{\text {lower }}$ : Lower threshold of the station.

(2) Auxiliary Decision Variables, $\forall t=1,2, \cdots, T$

$v m_{t, t^{\prime}}^{(+), v_{t}}$ : Binary variable (equals 1 when vehicle $v_{t}=1,2, \cdots, \delta_{t}^{(n+)}$ is required to be moved in at time step $t$ and actually arrives at time step $t^{\prime}$, otherwise 0 )

$v m_{t, t^{\prime}}^{(-), v_{t}}$ : Binary variable (equals 1 when vehicle $v_{t}=1,2, \cdots, \delta_{t}^{(n-)}$ is required to be moved out at time step $t$ and actually departures at time step $t^{\prime}$, otherwise 0 )

$v s_{t}^{b, b^{(D)}}$ : Binary variable (equals 1 when vehicle with battery status $b$ is matched with demand on battery status $b^{(D)}$, otherwise 0 ).

(3) Stochastic Variables, $\forall t=1,2, \cdots, T$

$r_{t}$ : The number of returned vehicles in time step $t$

$d_{t}^{(g e)}:$ The number of demand vehicles generated in time step $t$

$b_{t, v_{t}}^{(R)}$ : The battery status of each returned vehicle in time step $t, \mathrm{~km}$ (it follows a continuous distribution; $\left.b_{t}^{(R)} \in B_{t}^{(R)}, v_{t}=1,2, \cdots, r_{t}\right)$

$b_{t, v_{t}}^{(g e)}$ : The desired battery status of demands generated in time step $t, \mathrm{~km}$ (it follows a continuous distribution; $b_{t}^{(g e)} \in B_{t}^{(g e)}$, $v_{t}=1,2, \cdots, d_{t}^{(g e)}$ )

$b_{t, v_{t}}^{(+)}$: The battery status of each vehicle that is actually relocated into the station in time step $t, \mathrm{~km}$ (it follows a continuous distribution; $b_{t}^{(+)} \in B_{t}^{(+)}, v_{t}=1,2, \cdots, \delta_{t}^{(+)}$)

$b_{t, v_{t}}^{(-)}$: The desired battery status of each vehicle that is relocated out of the station in time step $t, \mathrm{~km}$. (it follows a continuous distribution; $b_{t}^{(-)} \in B_{t}^{(-)}$, $\left.v_{t}=1,2, \cdots, \delta_{t}^{*(-)}\right)$

$t_{v_{t}}^{(+)}$: Delay from the time step of the call of move-in to the time step of the arrival of $v_{t}$, due to the moving time consumption, following a discrete distribution $\left(v_{t}=1,2, \cdots, \delta_{t}^{(n+)}\right)$

$t_{v_{t}}^{(-)}$: Delay from the time step of the call of move-out to the time step of the departure of $v_{t}$, due to the availability of relocation staff, following a discrete distribution $\left(v_{t}=1,2, \cdots, \delta_{t}^{(n-)}\right)$

$p_{t}^{b}: \quad \forall b \in B_{t}^{D(u s)}$, equal to 1 if the rejected demands remain to the next time step and 0 if not, at the end of time step $t$. (c) Dependent Variables, $\forall t=1,2, \cdots, T$

$x_{t}$ : The number of remaining vehicles at the end of time step $t\left(x_{0}=0\right.$ for initial condition)

$d_{t}$ : The number of total demand vehicles in time step $t$

$d_{t}^{(s t)}$ : The number of demands that are satisfied in time step $t$

$\delta_{t}^{(n+)}$ : The number of vehicles needed to be relocated into the station in time step $t$ according to the threshold triggering

$\delta_{t}^{(n-)}$ : The number of vehicles needed to be relocated out of the station in time step $t$ according to the threshold triggering

$\delta_{t}^{(+)}$: The number of vehicles actually relocated into the station in time step $t$

$\delta_{t}^{(-)}$: The number of vehicles actually relocated out of the station in time step $t$ due to the battery status

$\delta_{t}^{*(-)}$ : The number of vehicles required to move out of the station in time step $t$ due to the delay

$u_{t}^{(+)}$: The time mark that there is no parking space available in time step $t\left(u_{t}^{(+)}=1, x_{t} \geq N ; 0\right.$, otherwise)

$u_{t}^{(-)}$: The time mark that there is no vehicle available in time step $t\left(u_{t}^{(-)}=1, x_{t} \leq 0 ; 0\right.$, otherwise $)$.

(d) Sets, $\forall t=1,2, \cdots, T$

$B_{t}^{(R)}$ : Battery status of returned vehicles in time step $t$

$B_{t}^{(g e)}$ : Desired battery status of each generated demand vehicle in time step $t$

$B_{t}^{(+)}$: Battery status of vehicles to be relocated in in time step $t$

$B_{t}^{(-)}$: Battery status of vehicles to be relocated out in time step $t$

$B_{t}^{V(r m)}$ : Battery status of the remaining vehicles at the end of time step $t, B_{t}^{V(r m)}=\emptyset$ for initial condition

$B_{t}^{D(u s)}$ : The unsatisfied demand battery status set at the end of time step $t$

$B_{t}^{D(r m)}$ : The remaining demand battery status set at the end of time step $t, B_{t}^{D(r m)}=\emptyset$ for initial condition.

\section{Data Availability}

The original data that supports this paper can be accessed from the website at https://drive.google.com/drive/folders/ 1qZOpGqHCG1dCtUlnMUlFRnPjuh5lp5sZ or obtained from the corresponding author upon request. 


\section{Conflicts of Interest}

The authors declare that there are no conflicts of interest regarding the publication of this paper.

\section{Acknowledgments}

Special thanks are due to Shanghai International Automobile City Co., Ltd., for the collaborative research projects of "Research and Application of the Key Technology of Electric Car Rental Sharing System Based on Internet of Vehicles" and "The Threshold Method of Vehicle Allocation Technology". The research is supported by the National Natural Science Foundation of China (no. 51722809), the National Key Research and Development Program of China (no. 2016YFE0206800), the Fok Ying-Tong Education Foundation (no. 151076), Humanity and Social Science Youth Foundation of Ministry of Education of China (no. 16YJCZH070), and Ministry of House and Urban-Rural Development of China Project (2015K5006).

\section{References}

[1] S. Shaheen, D. Sperling, and C. Wagner, Carsharing in Europe and North American: Past, Present, and Future, University of California Transportation Center, 2001.

[2] M. Barth, S. A. Shaheen, T. Fukuda, and A. Fukuda, "Carsharing and station cars in Asia: Overview of Japan and Singapore," Transportation Research Record, no. 1986, pp. 106-115, 2006.

[3] S. Shaheen, N. Chan, A. Bansal, and A. Cohen, Shared Mobility: A Sustainability Technologies Workshop: Definitions, Industry Developments, and Early Understanding, University of California, Berkeley, Transportation Sustainability Research Center; California Department of Transportation, 2015.

[4] A. Jung, "Fewer Cars, More Mobility: Can Carsharing Work in China?" International Transportation, vol. 67, no. 1, pp. 26-29, 2015.

[5] S. A. Shaheen, N. D. Chan, and H. Micheaux, "One-way carsharing's evolution and operator perspectives from the Americas," Transportation, vol. 42, no. 3, pp. 519-536, 2015.

[6] A. G. H. Kek, R. L. Cheu, and M. L. Chor, "Relocation simulation model for multiple-station shared-use vehicle systems," Transportation Research Record, no. 1986, pp. 81-88, 2006.

[7] A. G. H. Kek, R. L. Cheu, Q. Meng, and C. H. Fung, "A decision support system for vehicle relocation operations in carsharing systems," Transportation Research Part E: Logistics and Transportation Review, vol. 45, no. 1, pp. 149-158, 2009.

[8] J. Firnkorn and M. Müller, "What will be the environmental effects of new free-floating car-sharing systems? The case of car2go in Ulm," Ecological Economics, vol. 70, no. 8, pp. 15191528, 2011.

[9] M. Namazu and H. Dowlatabadi, "Characterizing the GHG emission impacts of carsharing: A case of Vancouver," Environmental Research Letters, vol. 10, no. 12, 2015.

[10] S. A. Shaheen, A. P. Cohen, and J. D. Roberts, "Carsharing in North America: Market growth, current developments, and future potential," Transportation Research Record, no. 1986, pp. 116-124, 2006.

[11] S. Le Vine, M. Lee-Gosselin, A. Sivakumar, and J. Polak, "A new approach to predict the market and impacts of round-trip and point-to-point carsharing systems: case study of London,"
Transportation Research Part D: Transport and Environment, vol. 32, pp. 218-229, 2014.

[12] K. M. N. Habib, C. Morency, M. T. Islam, and V. Grasset, "Modelling users' behaviour of a carsharing program: Application of a joint hazard and zero inflated dynamic ordered probability model," Transportation Research Part A: Policy and Practice, vol. 46, no. 2, pp. 241-254, 2012.

[13] C. Morency, K. M. N. Habib, V. Grasset, and M. T. Islam, "Understanding members' carsharing (activity) persistency by using econometric model," Journal of Advanced Transportation, vol. 46, no. 1, pp. 26-38, 2012.

[14] B. Boyaci, K. G. Zografos, and N. Geroliminis, "An optimization framework for the development of efficient one-way car-sharing systems," European Journal of Operational Research, vol. 240, no. 3, 2015.

[15] G. H. D. A. Correia and A. P. Antunes, "Optimization approach to depot location and trip selection in one-way carsharing systems," Transportation Research Part E: Logistics and Transportation Review, vol. 48, no. 1, pp. 233-247, 2012.

[16] M. Nourinejad and M. J. Roorda, "A dynamic carsharing decision support system," Transportation Research Part E: Logistics and Transportation Review, vol. 66, pp. 36-50, 2014.

[17] R. Zakaria, M. Dib, L. Moalic, and A. Caminada, "Car relocation for carsharing service: Comparison of CPLEX and greedy search," in Proceedings of the 2014 IEEE Symposium on Computational Intelligence in Vehicles and Transportation Systems, CIVTS 2014, pp. 51-58, USA, December 2014.

[18] A. Carlier, A. Munier-Kordon, and W. Klaudel, "Mathematical model for the study of relocation strategies in one-way carsharing systems," Transportation Research Procedia, vol. 10, pp. 374383, 2015.

[19] M. Bruglieri, A. Colorni, and A. Luè, "The relocation problem for the one-way electric vehicle sharing: An Application to the Milan Case," in Proceedings of the Social and Behavioral Sciences, vol. 111, pp. 18-27, 2014.

[20] M. Repoux, B. Boyaci, and N. Geroliminis, "Simulation and Optimization of One-Way Car-Sharing Systems with Variant Relocation Policies," in Transportation Research Board, 2015.

[21] A. Febbraro, N. Sacco, and M. Saeednia, "One-Way Carsharing: Solving the Relocation Problem," Transportation Research Record, no. 2319, pp. 113-120, 2012.

[22] G. Santos and G. Correia, "A MIP model to optimize real time maintenance and relocation operations in one-way carsharing systems," Transportation Research Procedia, vol. 10, pp. 384-392, 2015.

[23] M. Barth and M. Todd, "Simulation model performance analysis of a multiple station shared vehicle system," Transportation Research Part C: Emerging Technologies, vol. 7, no. 4, pp. 237259, 1999.

[24] E. M. Cepolina and A. Farina, "Urban car sharing:An overview of relocation strategies," in Proceedings of the 18th International Conference on Urban Transport and the Environment, UT12, pp. 419-431, Spain, May 2012.

[25] E. M. Cepolina and A. Farina, "A methodology for planning a new urban car sharing system with fully automated personal vehicles," European Transport Research Review, vol. 6, no. 2, pp. 191-204, 2014.

[26] E. M. Cepolina, A. Farina, C. Holloway, and N. Tyler, "Innovative strategies for urban car-sharing systems and a simulator to assess their performance," Transportation Planning and Technology, vol. 38, no. 4, pp. 375-391, 2015. 
[27] G. Cao, L. Wang, Y. Jin et al., "Determination of the vehicle relocation triggering threshold in electric car-sharing system," Lecture Notes in Electrical Engineering, vol. 404, pp. 11-22, 2016. 


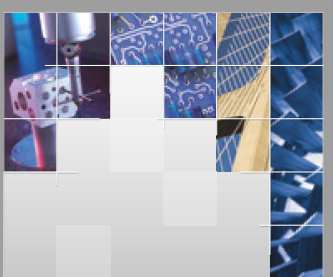

\section{Enfincering}
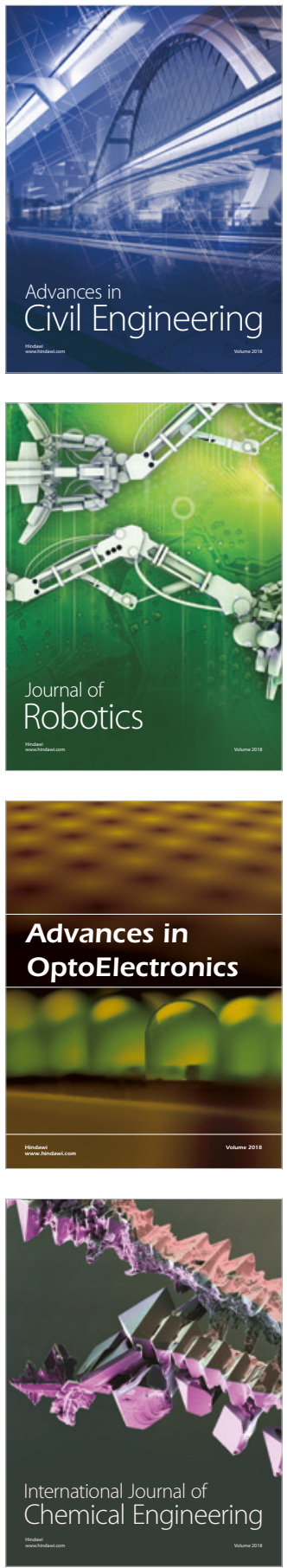

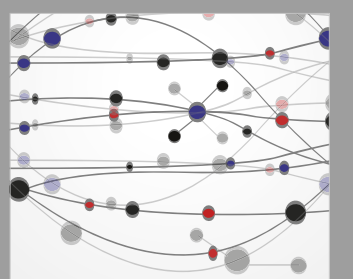

\section{Rotating \\ Machinery}

The Scientific World Journal

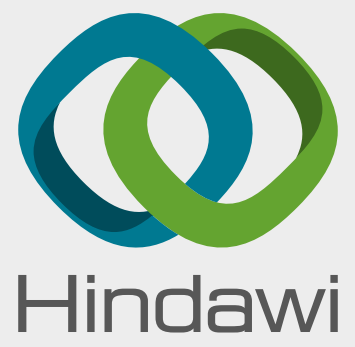

Submit your manuscripts at

www.hindawi.com
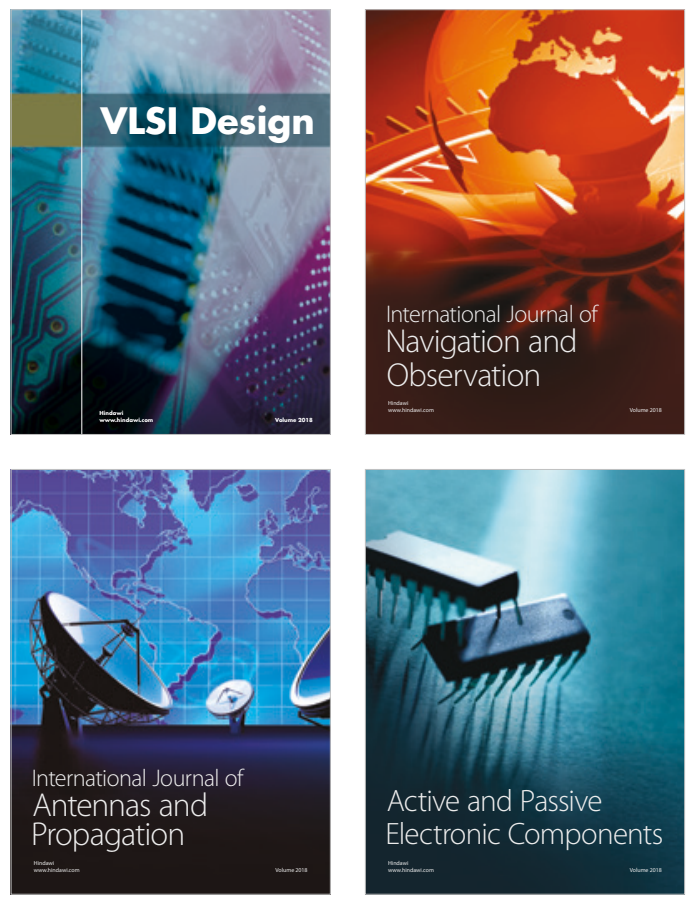
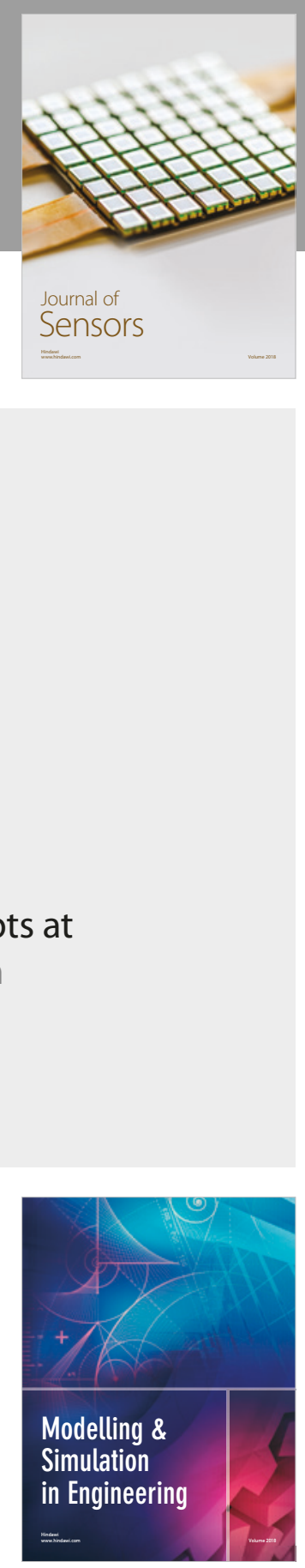

\section{Advances \\ Multimedia}
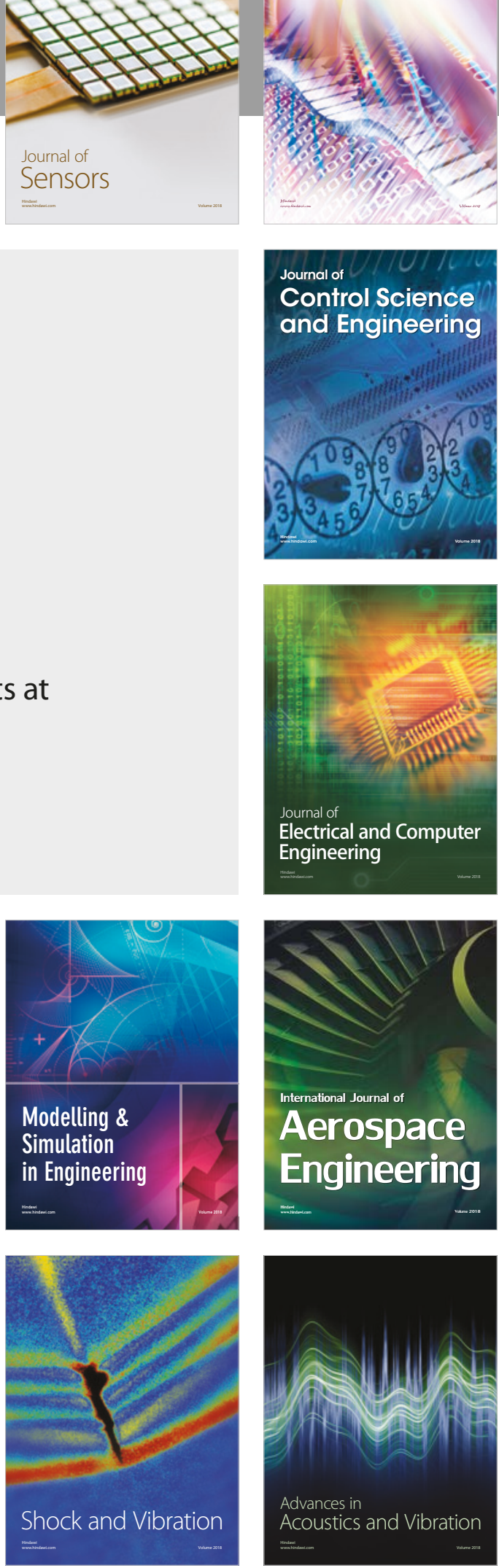